\title{
Fast Algorithms for Transport Models DE-FG02-90ER25086
}

\author{
Tom Manteuffel, Principal Investigator
}

April, 1992

\section{Report of Technical Progress}

The first two years of this project were mainly focused on developing fast algorithms for the solution of the equations arising from models of single group isotropic transport of neutrally charged particles in slab geometry. In this context, the equation governing transport is an integral-differential equation involving one space variable and one angle variable. The equation. for a semi-infinite slab of width $a$ is

$$
\mu \frac{\partial \psi}{\partial x}+\sigma_{t} \psi=\frac{\sigma_{s}}{2} \int_{-1}^{1} \psi\left(x, \mu^{\prime}\right) d \mu^{\prime}+q
$$

for $x \in(0, a)$ and $\mu \in(-1,1)$. Here $\psi$ is the flux of particles at the position $x$ traveling at an angle $\theta$ from the $x$-axis $(\mu=\cos (\theta))$. The problem is well-posed if boundary conditions give the flux entering the slab, that is,

$$
\begin{aligned}
& \psi(0, \mu)=g_{0}(\mu), \quad \text { for } \mu>0 \\
& \psi(a, \mu)=g_{a}(\mu), \quad \text { for } \mu<0 .
\end{aligned}
$$

This equation is normally discretized by a $S_{N}$ approximation, that is, by expanding the angular dependence in terms of the first $N$ Legendre prlynomials. Semi-discrete equations are formed by using a Galerkin formulation. The spatial dependence is then discretized by either finite difference or finite element methods.

The problem becomes difficult to solve when there is little absorption and the mean free path becomes small compared to the physical dimensions of the problem, that is, $\sigma_{t} \gg 1$ and $\gamma=\frac{\sigma_{2}}{\sigma_{t}} \sim 1$. This is called the optically dense limit. The limiting operator becomes singular, and especially so on the subspace containing smooth functions.

Numerically, this limit is determined by $\sigma_{t} h$ where $\sigma_{t} d x$ represents the collision probability cross-section and $h$ is the discrete cell width. In brief, $\frac{1}{\sigma_{t} h}$ represents the rumber of mean free paths per cell width. 
Classical algorithms for solving transport equations become prohibitively expensive in the optically dense limit. A recent improvement, based on the observation that isotropic scattering looks like diffusion in this limit, is called Diffusion Synthetic Acceleration (DSA) [1]. This method may be viewed as preconditioning the problem by the solution of a diffusion equation [5]. Typically, the diffusion equation is solved by using multigrid. DSA achieves a convergence factor of approximately .23 per iteration. Unfortunately, DSA does poorly on anisotropic problems, is very sensitive to the spatial difference scheme and is difficult to implement in higher dimensions.

This project has developed multigrid algorithms for the transport equations directly that are more efficient than DS.A and are easily generalized to anisotropic equations and higher dimensions. In the first year of this project a multigrid scheme was developed for the equations that result when an upwind difference scheme (UW) is used for the spatial dependence. In the optically dense limit, a v-cycle convergence factor of $\rho=O\left(\frac{1}{\sigma, h}\right)$ was obtained; that is, the algorithm converges faster as the optically dense limit is approached. Jnfortunately, UW yields a poor approximation to the differential equation in this limit. The Modified Linear Discontinuous Difference scheme (MLD) [6] is both second-order accurate and behaves properly in the optically dense limit. For this difference scheme, $v$-cycle convergence factcrs of .04-.1 were achieved. The algorithm used a relaxation that inverts along lines in the angle variable, called red/black $\mu$-line relaxation. This relaxation is very inexpensive because it takes advantage of the rank-one nature of the isntropic scattering operator. For large $\sigma_{t} h$ the UW scheme vras used as a coarse grid operator for MLD. Because of the speed at which the UW scheme can be solved for large $\sigma_{t} h$, the algorithm is very efficient. A paper announcing these results was included in the Proceedings of the IMACS First International. Conference on Computational Physics held in Boulder, Colorado, June 11-15, 1990 [7].

In the second year of this project a greatly improved multigrid scheme for the MLLI equations was developed. This scheme employs a 2-cell red/black $\mu$-line relaxation and improved restriction operators. It was implemented on both regular grids and highly irregular grids, or equivalently, highly heterogeneous material. For the case with no atsorption, that is, $\gamma=1$, the following theoretical bounds on the convergence factor for a $\mathrm{v}(1,1)$-cycle were established: 
- If $\max _{i}\left(\sigma_{t} h_{i}\right) \ll 1$, then $\rho \leq O\left(\left(\sigma_{t} h_{i}\right)^{2}\right)$.

- If $\max _{i}\left(\sigma_{t} h_{i}\right) \gg 1$, then $\mu \leq O\left(\frac{1}{\left(\sigma_{t} h_{i}\right)}\right)$.

Numerical results were even better and implied the following bounds:

- If $\max _{i}\left(\sigma_{t} h_{i}\right) \ll 1$, then $\rho \leq O\left(\left(\sigma_{t} h_{i}\right)^{3}\right)$.

- If $\max _{i}\left(\sigma_{t} h_{i}\right) \gg 1$, then $\rho \leq O\left(\frac{1}{\left(\sigma_{t} h_{i}\right)^{2}}\right)$.

Moreover, the slowest observes convergence occurred for grids on which $\sigma_{t} h_{\mathrm{i}} \sim 1$ and yielded convergence factor $\rho \sim 05$. These results have been summarized in the attached report [10].

It is important to note that for the $S_{2}$ approximation, one $\mathrm{v}$-cycle gave the exact solution. This is because, in this case, the v-cycle was actually performing block cyclic reduction.

Unfortunately, the results for $\gamma \neq 1$ were not entirely satisfactory. For $\gamma=$ $1-\frac{\alpha}{\left(\sigma_{t} h_{1}\right)^{p}}$ where $p \geq 3$, the algorithm performed at the theoretical rates above. For, $p \leq 1$, the $v$-cycle was almost an exact inversion, performing at least as fast as the theoretical rates. However, for $p=2$ the algorithm produced convergence factors as iarge as .4 in the dense limit. This is explained by the heuristic that if there is no absorption the solution is linear in the dense limit. Therefore, after 2-cell relaxation the error across each two cell pair is linear and, thus, in the range of the interpolation. If there is a lot of absorption, the error is almost zero and relaxation alone is sufficient to solve the problem. However, if there is just enough absorption, then after relaxation the error becomes a kinked linear across tivo cells.

With this heuristic, a modified interpolation was developed. The interpolation assumed that the arror would be a kinked linear across two cells. The size of the kink was determined by an asymptotic analysis. An operator induced interpolation was developed and the new algorithm produced rates on the same order as the theoretical convergence rates given above. The coarse grid operators that result from operator induced interpolation no longer resemble the fine grid operator transferred to the coarse grid. However, for this application the coarse grid operators retain the properties that permit fast 2-cell $\mu$-line relaxation. It is interesting to note that operator induced interpolation closely resembles cyclic reduction. Again, for $S_{2}$ the v-cycle is an exact inversion. A report on this new algorithm is being written and will comprise a portion of the thesis of Yang, Gaoming. 
Also during the second year of this project the multigrid algorithm without kinked elements was programmed on the Thinking Machines Inc. CM-2 located at Los Alamos National Labs. The red/black 2-cell $\mu$-line relaxation was carefully organized to take advantage of the SIMD architecture. Each. point on the computational grid was assinged a single processor. Evaluation of the terms corresponding to the integral requires data for all angles at each spatial point. The intrinsic function sum and prestoring of necessary data minimized the sequential nature of this portion of the algorithm. The resulting code produced solutions in an amount of time essentially independent of the size of the problem until the machine reached saturation. A report on this implementation will appear in the Proceedings of the Copper Mountain Conference on Iterative Methods, April 10-14, 1992 [11](attached)

\section{Future Plans}

In the coming year this project will move ahead on several fronts. The first goal is to implement a parallel version of the multigrid algorithm for anisotropic scattering in slab geometry. This will use the multilevel in angle algorithm developed by Morel and Manteuffel [13]. The portion of this algorithm that corresponds to a relaxation is the shifted transport sweep, which involves solving equations of the form

$$
\mu \frac{\partial \psi}{\partial x}+\left(\sigma_{t}-\alpha\right) \psi=Q
$$

with boundary conditions

$$
\begin{aligned}
& \psi(0, \mu)=g_{0}(\mu), \quad \text { for } \mu>0, \\
& \psi(a, \mu)=g_{a}(\mu), \quad \text { for } \mu<0 .
\end{aligned}
$$

Here $\mathrm{Q}$ includes the scattering source from a previous iteration.

On a serial machine one would integrate across the slab for each angle independently, starting at the left boundary for positive angles and at the right boundary for negative angles. At first glance, these equations are parallel in angle, but sequential in space. However, the integration can also be accomplished by performing cyclic reduction on the block lower (upper) triangular matrices associated with the positive (negative) angles. Preliminary analysis indicates that this approach is stable and easily implemented. Thus, in addition to complete independence in angle, the process will be partially parallel in space. Implementation of this algorithm on the CM-2 has already begun. 
In this algorithm, the coarsest level corresponds to an isotropic $S_{2}$ problem, which can be solved by a single v-cycle of the isotropic algorithm described above.

Another goal of this project is to generalize both the isotropic and anisotropic slab algorithms to two-dimensional problems with logically rectangular grids discretized by Linear Discontinuous (LD) elements. For isotropic problems we will first consider a multi-cell red/black relaxation and operator induced interpolation, as in the slab geometry. For anisotropic problems we will examine solving the transport sweeps with two dimensional cyclic reduction. Again the coarsest level will be an $S_{2}$ isotropic problem.

A third focus of this project will be the development of more robust difference schemes based on a least squares formulation, rather than the Galerkin formulation used in the LD and MLD discretization schemes. Since the spatial derivatives are first order, a least squares functional can be constructed that admits piecewise linear elements. 'The LD and MLD schemes attempt to deal with boundary layers at cell edges by introducing a discontinuity in the up wind direction. This is important in the optically dense limit. However, this can be better handled with exponentially fitted finite elements or optimal adjoint finite element methods (c.f. [2], [3]). A least squares functional will provide a simple framework for implementing multigrid and multilevel algorithrns (c.f. [12]).

Other Work Three other papers that were partially funded by this project are also attached. The first paper [8] is a result on the equivalence of discrete versions of elliptic second order operators. Specifically, it deals with the best self-adjoint preconditioning for a non-self-adjoint operator. The second paper [4] is a result on the numerical estimation of the diameter of a graph from the adjacency matrix and has application in designing advanced architecture computer networks. The final paper [9] is a study of the roots of the orthogonal and residual polynomials associated with a conjugate gradient iteration. It is shown that the roots may be used to construct possibly nonconvex regions in the complex plane that describe the spectrum of the operator.

\section{References}

[1] R. E. Alcouffe, "Diffusion Synthetic Acceleration Methods for 
the Diamond-Differenced Discrete-Ordinates Equations", Nucl. Sci. Eng., Vol. 64, p-344, 1977.

[2] E.T. Bouloutas and M.A. Celia, "An analysis of some classes of Petrov-Galerkin and optimal test function methods," Proc. 7th Intl. Conf. on Computational Methods in Water Resources, M.A. Celia et al. (eds.), Computational Mechanics Publications, Southampton, U.K., 1988, pp. 15-20.

[3] M.A. Celia, T.F. Russell, I. Herrera, and R.E. Ewirng, "An Eulerian-Lagrangian localized adjoint method for the advectiondiffusion equation," Advances in Water Resources, 13 (1990), 187-206.

[4] F.R.K. Chung, V. Faber and T. A. Manteuffel "An Upper Bound on the Diameter of a Graph from Eigenvalues Associated with its Laplacian," Los Alamos National Laboratories Report LAUR-91-2066, submitted to SIAM J. of Dis. Math., May, 1991. (attached)

[5] V. Faber and T. A. Manteuffel, "A Look at Transport Theory from the Viewpoint of Linear Algebra," Transport Theory, Invariant Imbedding and Integral Equations, Lecture Notes in Pure and Applied Mathematics Vol. 115 (Nelson, Faber, Manteuffel, Seth and White, eds.) Marcel-Dekker, April 1989.

[6] E. W. Larson and J. E. Morel, "Asymptotic Solution of Numerical Transport Problems in Optically-thick Diffusive Regimes II", J. Comp. Phys., Vol. 83, p-212, 1989.

[7] T. A. Manteuffel, S.F. McCormick, J. E. Morel and G. Yang, "Fast Multigrid Solver for Neutron Transport Problems," Proceedings of the IMACS First International Conference on Computational Physics, Boulder, Colorado, June 11 - 15, 1990.

[8] T. A. Manteuffel and J. S. Otto, "Optimal Equivalent Preconditionings," , submitted to SIAM J. of Numer. Anul. , February, 1991. (attached) 
[9] T. A. Manteuffel and J. S. Otto, "On the Roots of the Orthogonal Polynomials and Residual Polynomials Associated with a Conjugate Gradient Method," submitted to Joumal of Numerical Linear Algebra, March, 1992. (attached)

[10] T. A. Manteuffel, S.F. McCormick, J. E. Morel S. Oliveira and G. Yang, "Fast Multigrid Solver for Neutron Transport Problems," manuscript 1992. (attached)

[11] T. A. Manteuffel, S.F. McCormick, J. E. Morel S. Oliveira and G. Yang, "Parallel Multilevel Methods for Transport Equations", Proceedings of the 2nd Copper Mountain Conference on Iterative Methods, Copper Mountain, Colorado, April 10-14, 1992. (attached)

[12] S. F. McCormick, Multilevel Projection Methods for Partial Differential Equations, CBMS-NSF Regional Conference Series in Applied Math Vol. 62, SIAM Press, Philadelphia, 1991.

[13] J. E. Morel and T. A. Manteuffel, "An Angular Multigrid Acceleration Technique for $S_{n}$ Equations with Highly Forward-Peaked Scattering," Nucl. Sci. Eng.,, Vol. 107, pg 330, 1990.

\section{DISCLAIMER}

This report was prepared as an account of work sponsored by an agency of the United States Government. Neither the United States Government nor any agency thereof, nor any of their employees, makes any warranty, express or implied, or assumes any legal liability or responsibility for the accuracy, completeness, or usefulness of any information, upparatus, product, or process disclosed, or represents that its use would not infringe privately owned rights. Reference herein to any specific commercial product, process, or service by trade name, trademark, manufacturer, or otherwise does not necessarily constitute or imply its endorsement, recommendation, or favoring by the United States Government or any agency thereof. The vicws and opinions of authors expressed herein do not necessarily state or reflect those of the United States Government or any agency thereof. 

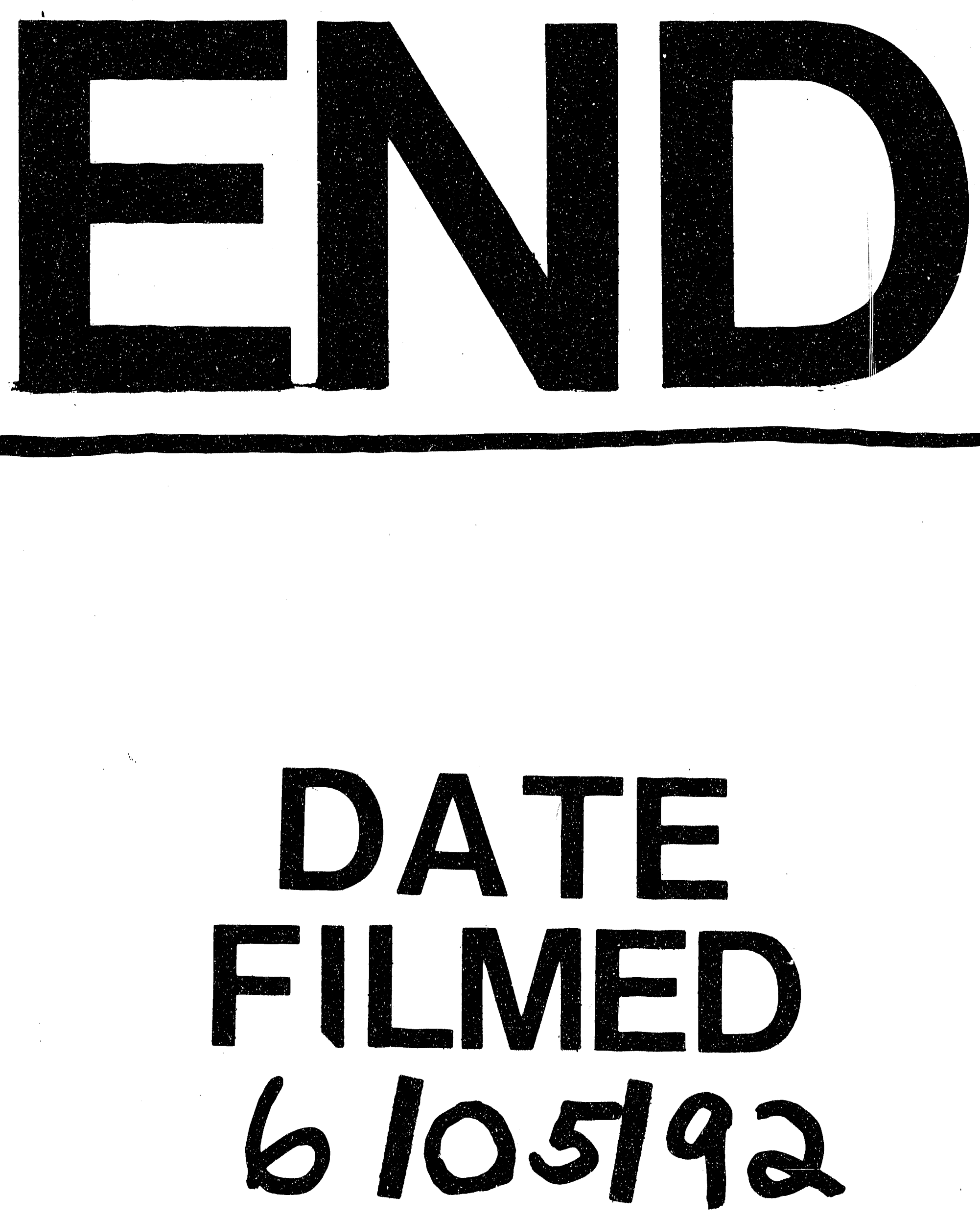

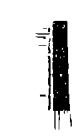


